\title{
The rhomboid flap in medial canthal reconstruction
}

\author{
S G J Ng, C F Inkster, B Leatherbarrow
}

\begin{abstract}
Aim-To describe a series of patients who have undergone a medial canthal reconstruction with a rhomboid flap.

Methods-A non-comparative interventional case series of 27 patients with medial canthal defects after Mohs excision of medial canthal basal cell carcinomas who underwent reconstruction using a rhomboid-shaped transpositional flap of adjoining skin and subcutaneous tissue. 25 cases were performed under local anaesthesia. The remaining two cases were combined with major lid reconstruction and performed under general anaesthesia. The outcome measures were closure of the defect, the cosmetic result, complications, and re-operations.

Results-Primary closure of the defect was achieved in all cases. The cosmetic result was highly satisfactory in all cases. There were no major complications or re-operations. Two cases had minor webbing of the medial upper lid.

Conclusions-The rhomboid flap is an effective, quick, and simple technique for medial canthal reconstruction. It provides excellent cosmesis and is associated with minimal complications. It can be modified according to the nature of the periorbital skin and the location, size, and depth of the defect.

(Br F Ophthalmol 2001;85:556-559)
\end{abstract}

The medial canthus is the second most common location for periorbital basal cell carcinomas. ${ }^{1}$ After tumour excision the resultant defect can be closed by a variety of methods depending on its size, location, depth, and patient preference. These include laissez faire, ${ }^{2}$ full thickness skin grafting, and various local flaps. ${ }^{3}$ The rhomboid flap was first described by Limberg in $1946 .{ }^{4}$ Its use in periocular skin defects has been described. ${ }^{35-7}$ This paper describes our experience of the rhomboid flap in patients with medial canthal defects following Mohs excision of basal cell carcinomas. We chose to use rhomboid flaps in medial canthal reconstruction because of their simplicity and versatility.

Hospital, Oxford

Road, Manchester

M13 9WH, UK

S G J Ng

C F Inkster

B Leatherbarrow

Correspondence to: Mr Brian Leatherbarrow bollin@mighty-micro.co.uk

Accepted for publication 20 December 2000 June 2000. The cases were collected prospectively. All patients had undergone Mohs excision of a basal cell carcinoma under local anaesthetic at another hospital no more than 24 hours before reconstructive surgery at our institution. Local anaesthetic without sedation was used except where a general anaesthetic was required for major lid reconstruction. Patients were treated on a day case basis except where general anaesthesia, social circumstances, or transport difficulties necessitated an inpatient stay. The reconstructive surgery and postoperative follow up were performed by one of the three authors. Data were recorded on the closure of the defect, the cosmetic result, complications, and re-operations.

\section{SURGICAL TECHNIQUE}

The defect is conceptualised as a rhomboid shape with its long axis vertical. The rhomboid consists of two equilateral triangles placed base to base (Fig 1A). For medial canthal defects there are two possible rhomboid flaps. These are constructed as follows. A line of the same length as the bases of the triangles is drawn horizontally across the nose from the base of the triangles. Two vertically oriented lines from the tip of the horizontal line are drawn at an angle of 60 degrees. These lines are the same length and parallel to the side of the rhomboid. The upper flap is used because of the greater laxity of the upper nasal skin. The resultant scar is also more easily hidden. The flap is oriented parallel to the lines of maximal extensibility (LME), allowing the donor site and defect to be closed with the minimum tension. (Fig $1 \mathrm{~B}$ and $\mathrm{C}$ ). The LME are perpendicular to the horizontally oriented relaxed skin tension lines (RSTL) on the bridge of the nose. The scar from closure of the flap's donor site is hidden in a RSTL. Despite three sections of the scar being oriented almost perpendicular to the RSTL (Fig 1D), in most cases it becomes insignificant within several weeks.

A suitable rhomboid flap is marked on the skin before the subcutaneous injection of bupivacaine $0.5 \%$ with 1:200 000 adrenaline. Firm manual pressure is applied for 5 minutes over the injected area to prevent haematomas. A blade is used to incise skin and subcutaneous tissue. The subcutaneous tissue at the base of the flap is undermined to enable it to stretch downwards into the defect. The edges of the defect are also undermined. Buried 5/0 Vicryl sutures are used to secure the flap in position. The undersurface of the flap is anchored to periosteum to reform the concave contour of the medial canthus. The nasal skin is closed with $6 / 0$ nylon vertical mattress sutures and the lid skin is closed with $7 / 0$ Vicryl sutures. A dental roll wrapped in paraffin gauze is applied under a firm pressure bandage. These measures help prevent haematomas and aid in 

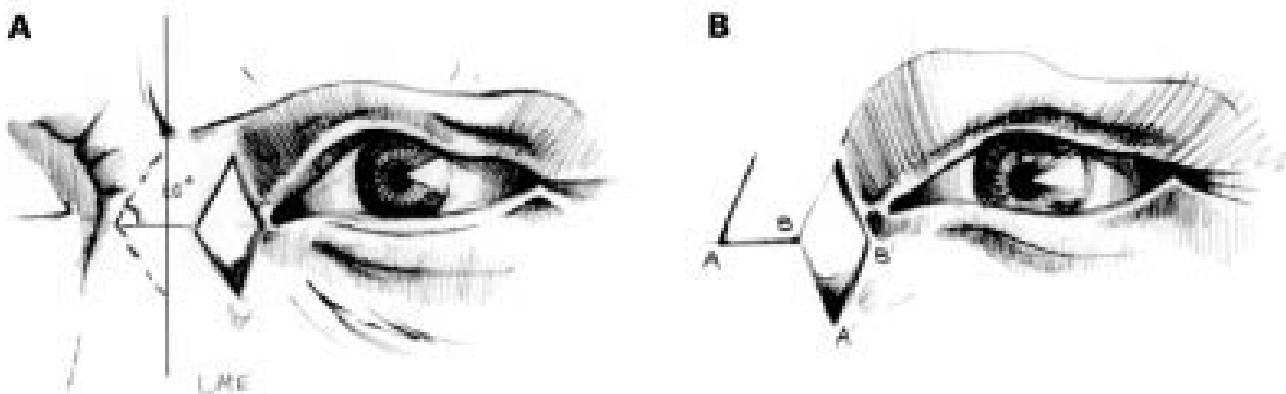

c
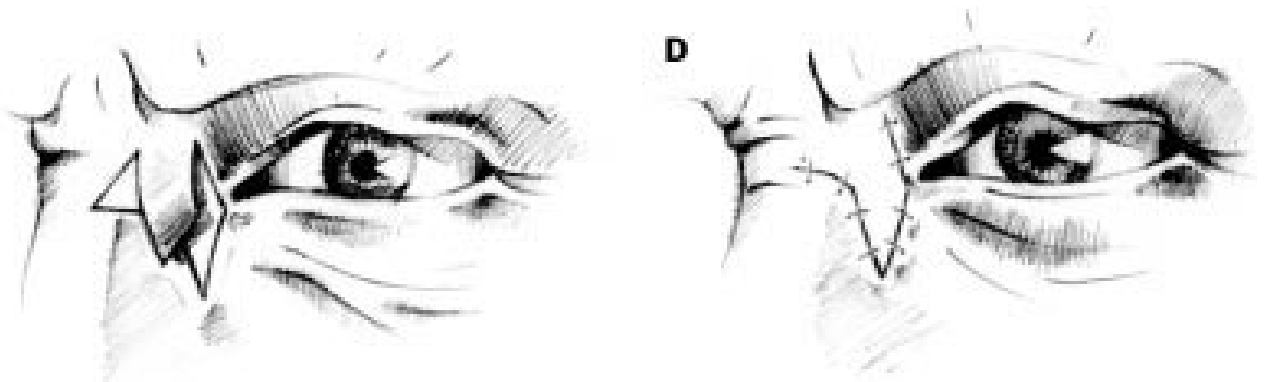

Figure 1 Rhomboid flap. (A) Construction of the two possible rhomboid flaps oriented parallel to the lines of maximum extensibility (LME). (B) Defect is closed by transposing points $A$ and $B$ to $A^{\prime}$ and $B^{\prime}$. (C) The upper flap closes the defect with the least distortion of surrounding tissues. (D) Resultant scar after suturing.

reforming the concave contour of the medial canthus. After at least 4 days the dressing is removed and the sutures are removed. Topical antibiotic ointment is applied for 1 week. Digital massage over the flap begins at suture removal and continues for at least 2 months.

Defects involving the areas above and below the medial palpebral ligament can be closed by rhomboid flaps from the adjoining glabellar and nasal tissues respectively. In closing inferiorly placed defects, the flap can be extended inferiorly by lengthening the vertical incision. In closing defects which extend laterally into the upper or lower lid, the lateral lid skin can be undermined and pulled medially to meet the rhomboid flap (Figs 2a and b). The orientation of the defect parallel to the LME minimises distortion on surrounding tissues. There is little risk of inducing medial ectropion (Figs 3a and $b$ ). When the medial palpebral ligament has been excised, periosteal flaps can be used to reattach the cut ends of the tarsal plates (Figs $4 \mathrm{a}$ and $\mathrm{b}$ ). A periosteal flap will pull the lids medially and helps reduce the size of the defect. Patients with thicker, more sebaceous skinless skin or younger patients with less skin laxity are at greater risk of postoperative wound

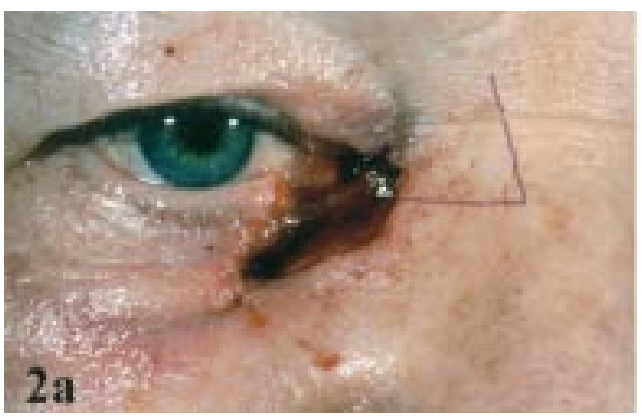

dehiscence and skin webbing. In such cases, more extensive undermining of the subcutaneous tissues and vigorous postoperative massage are recommended (Figs 5a and b).

\section{Results}

There were 27 patients with a mean age of 67.3 years. There were 16 males and 11 females. The mean follow up was 4 months. All but two cases were performed under local anaesthesia without sedation. Two cases were performed under general anaesthesia because of extensive defects involving the lower lid as well as the medial canthus (Table 1). Primary closure of the defect was achieved in all cases without wound dehiscence. In all cases the cosmetic appearance was highly satisfactory to the surgeons and patients. There were no intraoperative complications. Webbing of the medial upper lid area was present in two patients who had a large defect involving the glabellar region and the medial part of the upper lid. The webbing was reduced with vigorous massage and was not cosmetically problematic in either patient. There were no re-operations within the follow up period.

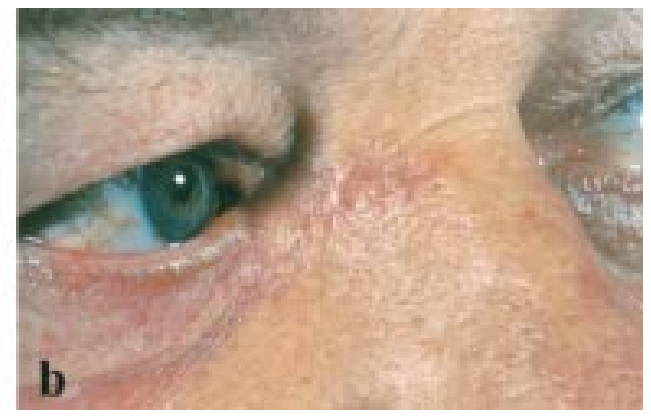

Figure 2 (a) Medial canthal defect extending into the lower lid with rhomboid flap marked. (b) Result 2 months postoperatively. 
Table 1 Patient characteristics and operation

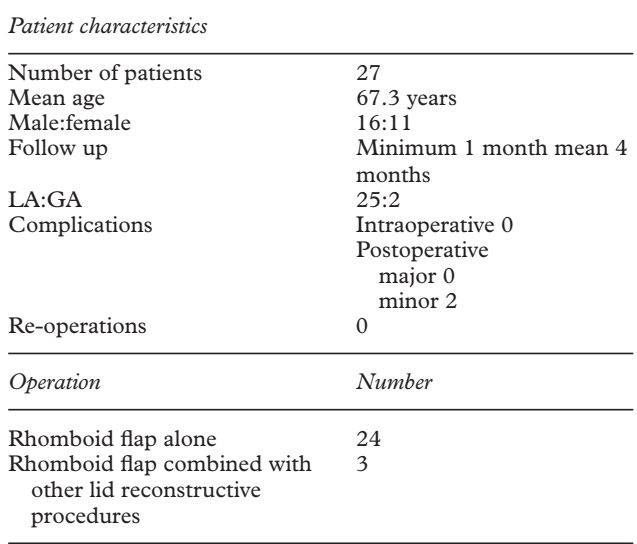

\section{Discussion}

Various techniques are used to repair medial canthal defects. The commonest methods are free full thickness skin grafts, rotational flaps - for example, the glabellar flap, ${ }^{8}$ bilobed
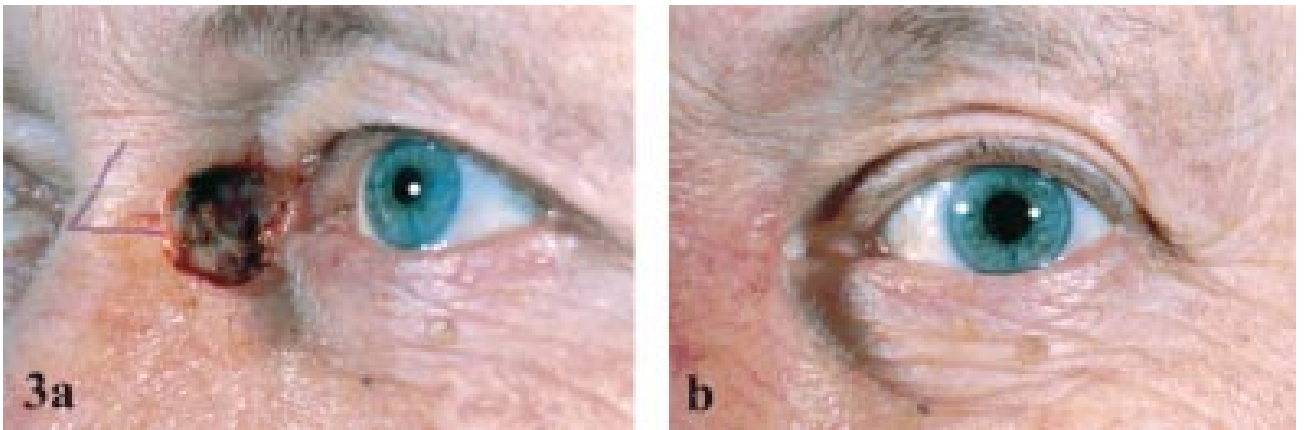

Figure 3 (a) Large medial canthal defect with rhomboid flap marked. (b) Minimal distortion of medial canthal tissues 3 months postoperatively.
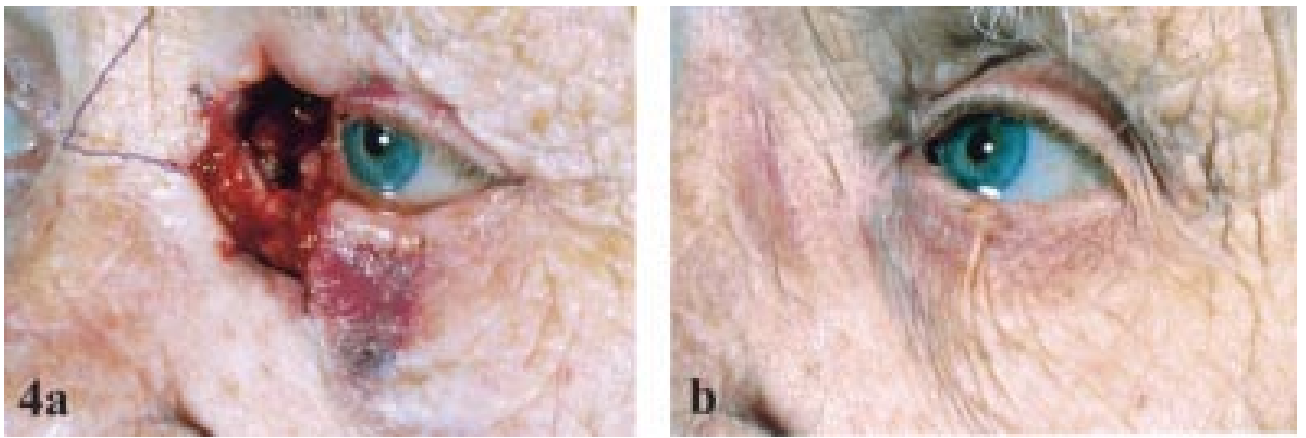

Figure 4 (a) Large medial canthal defect requiring periosteal and rhomboid flaps. (b) Result 5 months postoperatively (separate eyelid margin basal cell carcinoma removed at a later date).
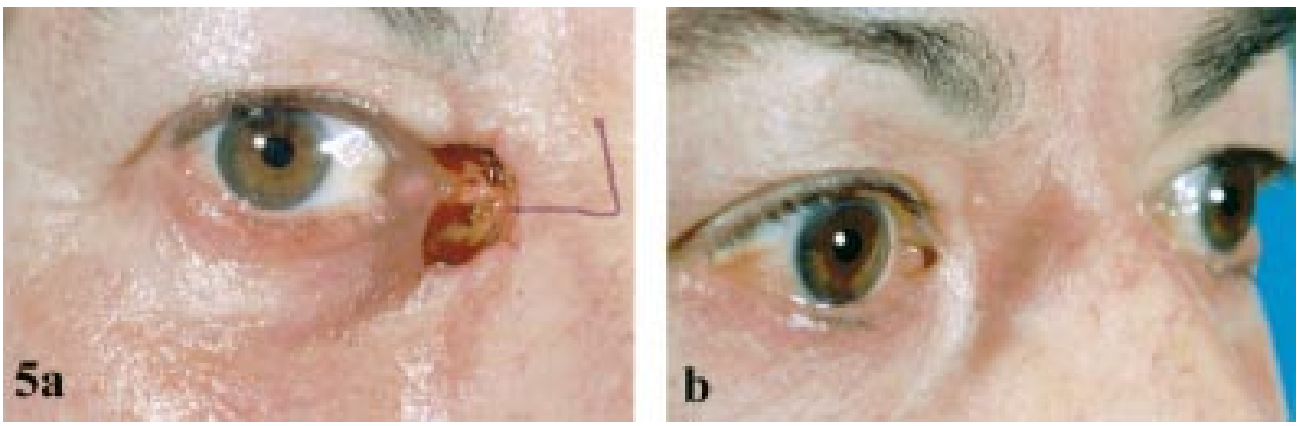

Figure 5 (a) Rhomboid flap for medial canthal defect in patient with thicker, sebaceous skin. (b) Result 9 months postoperatively. 
to older patients with thinner, less sebaceous skin. These individuals have greater skin laxity so that less traction is required to pull the flap into the defect. This series shows that excellent results can also be obtained in younger patients with less lax skin and patients with thicker sebaceous skin.

This study shows that for a wide variety of defects, the rhomboid flap is a simple yet effective technique for medial canthal reconstruction. It is minimally invasive, quick to perform, and suitable to be performed under local anaesthesia. It has minimal complications. It can be combined with other medial canthal and periocular reconstructive procedures such as periosteal flaps. Excellent cosmesis can be achieved. The flap's donor site is closed over the bridge of the nose. The resultant scar is hidden in a relaxed skin tension line. In the majority of cases the medial canthal flap becomes inconspicuous within a few weeks.

1 Inkster C, Ashworth J, Murdoch JR, et al. Oculoplastic reconstruction following Mohs surgery. Eye 1998;12:214-18.

2 Harrington JN. Reconstruction of the medial canthus by Harrington JN. Reconstruction of the medial canthus by
spontaneous granulation (laissez-faire): a review. Ann Ophspontaneous granulation
thalmol 1982;14:956-70.

3 Patrinely JR, Marines HM, Anderson RL. Skin flaps in periorbital reconstruction. Surv Ophthalmol 1987;31:249-61.

4 Limberg AA. Mathematical principles of local plastic procedures on the surface of the human body. Leningrad: Megriz, 1946. 5 Bullock JD, Koss N, Flagg SV. Rhomboid flap in ophthalmic plastic surgery. Arch Ophthalmol 1973;90:203-5.

6 Shotton FT. Optimal closure of medial canthal defects with defect orientations. Ophthalmic Surg 1983;14:46-52.

7 Borges AF. The rhombic flap. Plast Reconstruct Surg 1981;67:458-66.

8 Collin JRO. Eyelid reconstruction and tumour management. In: Manual of systematic eyelid surgery. 2nd ed. ment. In: Manual of systematic eyelid sure

9 Sullivan TJ, Bray LC. The bilobed flap in medial canthal Sullivan TJ, Bray LC. The bilobed flap in medial ca
reconstruction. Aust NZ $\mathcal{F}$ Ophthalmol 1995;23:42-8.

10 Bosniak SL, Zilkha MC. Ectropion. In: Nesi FA, Lisman RD, Levine MR eds. Smith's ophthalmic, plastic and reconstructive surgery. 2nd ed. St Louis: Mosby, 1987:290-307. 